\title{
Zygomaticomaxillary suture maturation: Part II-The influence of sutural maturation on the response to maxillary protraction
}

\author{
F. Angelieri ${ }^{1,2}$ (i) | A. C. Ruellas ${ }^{2,3}$ \\ M. S. Yatabe ${ }^{4}$ \\ L. H. S. Cevidanes ${ }^{2}$ \\ L. Franchi ${ }^{2,5}$ \\ C. Toyama-Hino ${ }^{6}$ \\ H. J. De Clerck ${ }^{7,8}$ \\ T. Nguyen ${ }^{7}$ \\ J. A. McNamara Jr',
}

\author{
${ }^{1}$ Department of Orthodontics, Guarulhos \\ University, Guarulhos, Brazil \\ ${ }^{2}$ Department of Orthodontics and Pediatric \\ Dentistry, School of Dentistry, The University \\ of Michigan, Ann Arbor, MI, USA \\ ${ }^{3}$ Department of Orthodontics and Pediatric \\ Dentistry, School of Dentistry, Federal \\ University of Rio de Janeiro, Rio de Janeiro, \\ Brazil \\ ${ }^{4}$ Bauru Dental School, University of São Paulo, \\ Bauru, Brazil \\ ${ }^{5}$ Department of Surgery and Translational \\ Medicine, The University of Florence, \\ Florence, Italy \\ ${ }^{6}$ Special Studies in Orthodontics, São Paulo \\ Military Hospital, São Paulo, Brazil \\ ${ }^{7}$ Department of Orthodontics, School of \\ Dentistry, University of North Carolina, \\ Chapel Hill, NC, USA \\ ${ }^{8}$ Private Practice, Brussels, Belgium \\ ${ }^{9}$ Center of Human Growth and \\ Development, School of Medicine, The \\ University of Michigan, Ann Arbor, MI, USA \\ Correspondence \\ F. Angelieri, Programa de Pós-Graduação \\ em Odontologia, Praça Tereza Cristina, 229 \\ Guarulhos, SP 07023-070, Brazil. \\ Email: fernandaang@yahoo.com.br
}

\begin{abstract}
Structured Abstract
Objective: To evaluate the influence of the maturational stages of zygomaticomaxillary sutures (ZMS) on the response to maxillary protraction.

Subjects and Methods: A total of 40 Class III patients were treated retrospectively with either a combination of rapid maxillary expansion and facial mask (RME/FM) or bone-anchored maxillary protraction (BAMP). The RME/FM group consisted of 18 patients (mean age 8.3 years), while the BAMP group was comprised of 22 patients (mean age 11.8 years). The initial CBCT images (T1) of the ZMSs were classified blindly. $3 \mathrm{D}$ models from $\mathrm{CBCT}$ images at the start and at the end of orthopaedic treatment were registered on the anterior cranial base, and corresponding structures were measured on colour-coded maps and semitransparent overlays. The amounts of protraction of the maxilla, zygoma, orbitale and maxillary first molars for both groups were analysed with two-way ANOVA with Holm-Sidak post hoc test for multiple comparisons. Results: A significant association was found between the early maturation stages of the ZMSs and the amount of maxillary protraction, regardless of the protraction method used. Class III patients with ZMS stages A and B showed greater maxillary protraction than patients at stage $\mathrm{C}$.

Conclusion: The maturational stages of ZMS are associated with the response maxillary protraction.
\end{abstract}

\section{KEYWORDS}

angle class III, cranial sutures, malocclusion, maxillary retrognathia

\section{1 | INTRODUCTION}

Rapid maxillary expansion and facial mask (RME/FM) therapy typically is indicated for treatment of Class III malocclusion associated with maxillary skeletal retrusion. ${ }^{1-4}$ The effects produced by this treatment protocol include more convexity of the facial profile ${ }^{5}$ due to forward displacement of the maxilla, ${ }^{4}$ clockwise rotation of the mandible, ${ }^{5}$ proclination of the maxillary incisors ${ }^{6}$ and a counterclockwise rotation of the palatal plane. ${ }^{7}$

To produce greater skeletal effects, early RME/FM treatment has been advocated. However, different chronological ages have been proposed as the best timing for initiating the RME/FM protocol. While some authors have recommended treatment up to 8 years, ${ }^{8} 9$ years 9 and 10 years of age ${ }^{10,11}$ or before puberty, ${ }^{12}$ others did not identify any differences in response according to chronological age., 2,13,14

Great individual variability in the amount of maxillary protraction has been demonstrated also in patients treated with bone-anchored maxillary protraction (BAMP). ${ }^{15}$ BAMP is initiated in the late mixed dentition or early permanent dentition. De Clerck and co-workers state that the placement of the miniplates typically should be delayed until after 10 years of age because the zygomatic plates tend to fail more often in younger patients. ${ }^{16}$

The amount of maxillary protraction is dependent on the maturation of the circummaxillary sutures, including the transverse palatine 
suture, the frontomaxillary sutures and the zygomaticomaxillary sutures (ZMSs). Kambara ${ }^{17}$ demonstrated that the ZMSs presented similar histological findings, or even greater complexity of interdigitations, compared to other circummaxillary sutures in young (mixed dentition phase) and older (permanent dentition phase) monkeys. The ZMSs are the longest and thickest circummaxillary sutures. ${ }^{18-20}$ They are oriented along the direction of the applied force system used in maxillary protraction. ${ }^{21}$ Individual assessment of the maturation of ZMSs, therefore, may represent an indicator of the response to orthopaedic maxillary protraction.

In Part 1 of this investigation, ${ }^{22}$ we presented a classification of the maturational stages of the ZMSs. The aim of this study was to evaluate the influence of the maturational stages of the ZMSs on the amount of maxillary protraction produced by RME/FM and BAMP protocols in growing patients with Class III malocclusion. The differences between the two protocols were of secondary interest because the main goal of this study was to evaluate an adequate sample of children who underwent maxillary protraction at the three earliest stages of ZMS maturation and for whom $\mathrm{CBCT}$ data were available.

\section{2 | SUBJECTS AND METHODS}

The study sample was comprised of 45 Caucasian patients with Class III malocclusion diagnosed by a Wits appraisal of $-1 \mathrm{~mm}$ or less, an anterior cross-bite or incisor end-to-end relationship and a Class III molar relationship or mesial terminal step for the deciduous second molars. This retrospective study was approved by the Institutional Review Board from the University of Michigan.

Patients were treated with either RME/FM or BAMP protocols. The RME/FM group consisted of 18 patients (15 females, three males) with a mean age of 8.3 years (range from 5.6 to 10.7 years), treated consecutively by one orthodontist (C.T.H.) at the Methodist University of São Paulo in São Bernardo do Campo, Brazil. Rapid maxillary expansion was accomplished by way of a banded Hyrax expander, to which hooks were attached for elastics. Immediately after expansion, maxillary protraction was performed with an individual facial mask ${ }^{23}$ combined with extraoral elastics delivering 600 to $800 \mathrm{~g}$ of force per side. Patients were asked to wear the facial mask for 14 to 16 hours per day. The elastics were oriented from the expander to the facial mask in a downward and forward direction at an angle of 15 to 30 degrees relative to the occlusal plane. ${ }^{24}$

The BAMP group was comprised of 22 patients (12 females and 10 males) with a mean age of 11.8 years (range from 9.7 to 13.6 years), treated consecutively by one operator (H.D.C.), in a private practice in Brussels, Belgium. For the BAMP protocol, four miniplates (Bollard; Tita-Link, Brussels, Belgium) were inserted into the infrazygomatic crests of the maxillary buttress and between mandibular lateral incisors and canines. Extensions of these plates perforated the attached gingiva near the mucogingival junction. The initial force was 100g/ side, progressing to a maximum force of $250 \mathrm{~g} / \mathrm{side}$. Patients were instructed to wear the elastics $24 \mathrm{~h}$ /day. For patients who presented with an anterior cross-bite, a removable maxillary biteplate was placed to eliminate the occlusal interference until the overjet was corrected. ${ }^{16}$ Patients of both groups were treated at least to a positive overjet, with most patients overcorrected to a Class II molar relationship.

Both samples were analysed in a previous study in $2013 .^{24}$ For the current study, two male and two female patients were excluded from the RME/FM group due to poor image quality (artefacts) in the ZMS region that did not allow a reliable evaluation of the maturational stage. One male patient was replaced with a female patient, as this girl started her treatment later than others; therefore, the final RME/ FM sample consisted of 18 patients (15 females and three males). For the same reason, one female and two males were excluded from the original BAMP group as reported in 2013. ${ }^{24}$

Cone-beam computed tomography (CBCT) scans were obtained from all patients using an $\mathrm{CCAT}^{\mathrm{TM}}$ Cone Beam 3-D Imaging System (Imaging Science International, Hatfield, PA, USA). Each subject was seated in an upright position with the Frankfort plane (superior aspect of the external auditory canal to infraorbital rim line) parallel to the floor during the scanning process. For all scans, the minimum field of view (FOV) used was $16 \times 22 \mathrm{~cm}$, and the scan time was $20-40$ seconds. CBCT images were taken at the onset (T1) and after approximately 10 months for the RME/FM group and after 12 months for the BAMP group (T2).

\section{1 | Classification of the ZMSs maturation}

The CBCT images of the ZMSs at T1 were analysed using Invivo $5^{\mathrm{TM}}$ software (Anatomage, San Jose, CA, USA). Head orientation in the three planes of space and the selection of the slice for evaluation of the ZMSs maturation were performed according to the protocol described previously in the Part I of this study. ${ }^{22}$

The maturation of the ZMSs was determined in the sagittal view, at the infraorbital (superior) and infrazygomatic (inferior) portions of the suture. Radiographic interpretation was performed in the sagittal cross-sectional slice that best allowed visualization of the long axis of the ZMS. No adjustments in contrast or brightness of these images were performed. In a darkened room, the blinded ZMS samples were classified by one expert examiner (F.A.), according to the visual analysis method described in Part I of this study. Usually, the ZMSs of both sides presented the same maturational stage. Thus, for staging purpose, only one maturational stage of ZMSs for each patient was determined. Only two patients (one per group) showed different maturational stages of ZMSs on the right and left sides. For these cases, the more matured maturational stage of ZMSs was evaluated.

\subsection{Construction of 3D-surface models}

To evaluate the forward displacement of the maxilla and zygomas promoted by facial mask or BAMP treatments, 3D-surface models were constructed from CBCT images of all patients at T1 and T2. Model construction, cranial base registration and visualization, and assessment of treatment outcomes were performed according to protocols described in detail in previous studies. ${ }^{16,24,25}$

After acquisition, the CBCT scans were reformatted to an isotropic resolution of $0.5 \times 0.5 \times 0.5 \mathrm{~mm}$ to decrease the computational power 
and the time required to compute the automated registration. Threedimensional surface models of the anatomic region of interest were constructed using ITK-SNAP (open-source software; http://www. itksnap.org). The initial and final 3D models were registered on anterior cranial fossa structures (3D Slicer, open-source software, https:// www.slicer.org), specifically the endocranial surfaces of the cribriform plate region of the ethmoid bone and the internal surface of the frontal bone, as these regions had completed their growth early.

The 3DSlicer software was used to orient and measure maxillary displacement of the 3D surface models in both groups. ${ }^{26}$ Landmarks were placed to quantify T2-T1 changes: Point A (the most anterior point in the convexity of maxilla in the median sagittal plane), centre of the clinical crown of the right maxillary permanent central incisor, right and left most inferior point of the lower border of the orbits, right and left infraorbital foramen, right and left most inferior and posterior point of the zygomas, right and left mesial-buccal cusp of the maxillary permanent first molar. For statistical purposes, right and left mean values were utilized. The T2-T1 changes in Point A, and zygoma average, orbitale average, and first molar average were measured in the anteroposterior, supero-inferior and 3D directions.

\section{3 | Method error}

Twenty images of the ZMSs were selected randomly from the total sample and reclassified by the same examiner (F.A.) a month later. A $100 \%$ intra-examiner agreement was found.

Regarding the reliability of 3D-surface model measurements, 10 randomly selected patients were remeasured by the same observer. Intraclass correlation coefficient with a confidence level of $95 \%$ was performed to assess the reproducibility of the measurements between $\mathrm{T} 1$ and T2. The reliability of the segmentation and superimposition methods has already been validated. ${ }^{26-29}$

\section{4 | Statistical analysis}

Chi-square tests with Yates' correction were used to assess betweengroup differences in gender distribution. The influence of the maturation of ZMSs and the type of therapy (BAMP versus RME/FM) on the amount of three-dimensional displacements of the maxilla and upper permanent first molars (antero-posterior, supero-inferior and 3D displacement measured at Point A, zygoma average, orbitale average and first molar average) was evaluated by two-way ANOVA with Holm-Sidak post hoc test for multiple comparisons $(P<.05)$. The two-way ANOVA was performed on the T2-T1 changes for the threedimensional variables when both normal distribution (Shapiro-Wilk test) and equality of variances (Levene's test) were satisfied.

The power of the study was 0.82 (G*Power), ${ }^{30}$; it was calculated for two-way ANOVA for an effect size $\mathrm{f}$ of 0.55 for the three-dimensional displacement of the maxilla ${ }^{24}$ and a alpha value of 0.05 . The statistical analyses were performed with statistical software packages (SPSS 12, SPSS Inc., Chicago, Illinois, USA; SigmaStat 3.5, Systat software, Point Richmond, CA).

\section{3 | RESULTS}

The intraclass correlation coefficients (ICC) showed very good intraexaminer agreement varying from 0.79 to 1.00 for all measurements. Evaluation of ZMS maturation in both the RME/FM and BAMP groups is shown in Table 1. Only three cases (one female and two males) of the BAMP group showed the more mature stages $D$ and $E$. In the statistical analysis, we decided to exclude these three cases in order to have two treatment groups that were homogenous in terms of ZMS maturation stages. Thus, the BAMP group analysed statistically consisted of 19 patients (11 females and eight males).

No significant between-group differences were found in gender distribution (chi-square test=1.775; $P=.183$ ).

Preliminary evaluation of the data showed that each dependent variable (T2-T1 changes for the antero-posterior, supero-inferior and 3D directions of Point $A$, zygoma average, orbitale average and first molar average) was distributed normally (Kolmogorov-Smirnov test) for each combination of the groups for the two independent variables (ZMS maturation stage and type of therapy). The only exception was 3D direction for the first molars that was not distributed normally. For this variable, two-way ANOVA was not performed. Homogeneity of variances for each combination of the groups for the two independent variables also was assessed (Levene's test).

The descriptive data of the T2-T1 changes in the antero-posterior, supero-inferior and 3D directions for Point $A$, zygoma average, orbitale average and first molar average according to the different ZMS stages and type of therapy (BAMP and RME/FM) are shown in Tables 2 and 3 , respectively.

The antero-posterior displacement of Point A was influenced significantly by both the maturational stage of ZMSs and the type of therapy (Table 4). The forward displacement of the maxilla was significantly greater at ZMS maturational stage A and B compared to stage

TAB LE 1 Distribution of maturational stages of ZMS in RME/FM and BAMP groups

\begin{tabular}{|c|c|c|c|c|c|c|c|c|c|c|c|c|}
\hline \multirow[b]{3}{*}{ Stages } & \multicolumn{6}{|c|}{ RME/FM Group } & \multicolumn{6}{|c|}{ BAMP Group } \\
\hline & \multicolumn{2}{|l|}{ Gender } & \multicolumn{4}{|c|}{ Age (y) } & \multicolumn{2}{|l|}{ Gender } & \multicolumn{4}{|c|}{ Age (y) } \\
\hline & Female & Male & Mean & SD & Min & Max & Female & Male & Mean & SD & Min & Max \\
\hline$A$ & 4 & 1 & 7.5 & 1.3 & 5.7 & 8.5 & 3 & 2 & 11.5 & 1.8 & 9.7 & 13.6 \\
\hline$B$ & 9 & 1 & 8.2 & 1.6 & 5.6 & 10.2 & 6 & 4 & 11.9 & 1.1 & 10.0 & 13.6 \\
\hline C & 4 & 1 & 9.6 & 1.4 & 7.7 & 10.7 & 3 & 4 & 12.0 & 1.0 & 11.0 & 13.6 \\
\hline
\end{tabular}


TAB LE 2 Descriptive statistics for the three-dimensional T2-T1 changes according to the different ZMS stages $(\mathrm{mm})$

\begin{tabular}{|c|c|c|c|c|c|c|}
\hline & \multicolumn{2}{|c|}{$\begin{array}{l}\text { ZMS stage A } \\
(\mathrm{N}=9)\end{array}$} & \multicolumn{2}{|c|}{$\begin{array}{l}\text { ZMS stage } B \\
(\mathrm{~N}=18)\end{array}$} & \multicolumn{2}{|c|}{$\begin{array}{l}\text { ZMS stage C } \\
(\mathrm{N}=10)\end{array}$} \\
\hline & Mean & SD & Mean & SD & Mean & SD \\
\hline \multicolumn{7}{|c|}{ A point } \\
\hline$A-P$ & 2.7 & 1.6 & 2.5 & 1.6 & 1.3 & 0.8 \\
\hline S-I & 0.9 & 1.7 & 0.9 & 1.4 & 1.8 & 1.1 \\
\hline $3 D$ & 3.5 & 1.2 & 3.3 & 1.2 & 2.5 & 1.0 \\
\hline \multicolumn{7}{|c|}{ Average zygoma } \\
\hline$A-P$ & 2.0 & 1.2 & 1.7 & 1.2 & 1.2 & 0.7 \\
\hline S-I & 1.2 & 0.8 & 1.1 & 0.9 & 1.1 & 1.3 \\
\hline $3 \mathrm{D}$ & 2.6 & 1.2 & 2.7 & 0.7 & 2.2 & 0.9 \\
\hline \multicolumn{7}{|c|}{ Average orbitale } \\
\hline$A-P$ & 1.4 & 1.1 & 1.1 & 1.0 & 0.8 & 0.6 \\
\hline S-I & 0.4 & 0.6 & 0.2 & 1.1 & 0.6 & 0.8 \\
\hline $3 \mathrm{D}$ & 1.9 & 0.9 & 2.0 & 0.6 & 1.4 & 0.8 \\
\hline \multicolumn{7}{|c|}{ Average first molar } \\
\hline A-P & 3.7 & 2.3 & 3.7 & 2.0 & 2.3 & 0.8 \\
\hline S-I & 2.3 & 1.2 & 2.7 & 1.4 & 2.2 & 1.9 \\
\hline $3 D$ & 5.2 & 1.2 & 5.3 & 1.5 & 3.8 & 1.3 \\
\hline
\end{tabular}

SD, Standard deviation; A-P, antero-posterior; S-I, supero-inferior.

TABLE 3 Descriptive statistics for the three-dimensional T2-T1 changes according to the two treatment protocols $(\mathrm{mm})$

\begin{tabular}{|c|c|c|c|c|}
\hline & \multicolumn{2}{|c|}{ BAMP group $(\mathrm{N}=19)$} & \multicolumn{2}{|c|}{$\begin{array}{l}\text { RME/FM group } \\
(\mathrm{N}=18)\end{array}$} \\
\hline & Mean & SD & Mean & SD \\
\hline \multicolumn{5}{|l|}{ A point } \\
\hline$A-P$ & 3.1 & 1.2 & 1.3 & 1.2 \\
\hline S-I & 0.6 & 1.6 & 1.7 & 1.0 \\
\hline $3 \mathrm{D}$ & 3.7 & 1.2 & 2.6 & 0.9 \\
\hline \multicolumn{5}{|c|}{ Average zygoma } \\
\hline$A-P$ & 2.1 & 1.1 & 1.1 & 0.8 \\
\hline S-I & 1.2 & 1.0 & 1.0 & 1.0 \\
\hline $3 \mathrm{D}$ & 2.9 & 0.9 & 2.1 & 0.5 \\
\hline \multicolumn{5}{|c|}{ Average orbitale } \\
\hline$A-P$ & 1.6 & 0.9 & 0.6 & 0.6 \\
\hline S-I & 0.3 & 0.9 & 0.5 & 0.9 \\
\hline $3 \mathrm{D}$ & 2.1 & 0.8 & 1.5 & 0.6 \\
\hline \multicolumn{5}{|c|}{ Average first molar } \\
\hline$A-P$ & 3.8 & 1.5 & 2.9 & 2.2 \\
\hline S-I & 2.3 & 1.2 & 2.7 & 1.7 \\
\hline $3 D$ & 4.7 & 1.4 & 4.9 & 1.7 \\
\hline
\end{tabular}

SD, Standard deviation; A-P, antero-posterior; S-I,supero-inferior.

C (+1.3 mm and $1.4 \mathrm{~mm}$, respectively). As for the type of therapy, the BAMP group demonstrated significantly greater forward displacement of the maxilla $(+1.9 \mathrm{~mm}$ ) compared to RME/FM treatment (Table 3).
However, the interaction between ZMS maturational stages and type of therapy produced no significant effects on the antero-posterior displacement of Point A (Table 4).

The supero-inferior displacement of Point A was influenced significantly by the type of therapy, with the RME/FM group showing a significantly greater downward displacement of Point $A$ than the BAMP group (1.2 mm, Table 4). The 3D displacement of Point A was influenced significantly by the type of therapy, with the BAMP group exhibiting a significantly greater 3D displacement than the RME/FM group $(1.2 \mathrm{~mm})$. As for the average antero-posterior displacement of the zygomas, only the type of therapy had a significant influence (Table 5). The BAMP group exhibited a significantly greater forward displacement of the zygomas than did the RME/FM group $(1.0 \mathrm{~mm})$. The 3D displacement of the zygomas was influenced significantly by the type of therapy, with the BAMP group presenting a significantly greater 3D displacement than the RME/FM group $(0.9 \mathrm{~mm})$. No significant influence of either type of therapy or maturational stage of ZMSs was assessed for the supero-inferior displacement of the zygomas.

The antero-posterior displacement of orbits at orbitale was influenced significantly by the type of therapy and by the interaction between ZMS maturational stages and type of treatment (Table 6). The BAMP group showed a significantly greater forward displacement of the orbits with respect to RME/FM group $(1.2 \mathrm{~mm})$. Within both ZMS maturational stages $A$ and $B$, the BAMP group exhibited significantly greater forward displacement of the orbits with respect to RME/FM group $(2.0 \mathrm{~mm}$ and $1.0 \mathrm{~mm}$, respectively). No significant difference could be recorded between the 2 types of treatment within ZMS maturational stage $C$. When analysing the influence of the ZMS maturational stages within the BAMP group, only patients treated at stage $A$ showed a significantly greater forward displacement of the orbits than did patients treated at stage $C(1.3 \mathrm{~mm})$.

Within the RME/FM group, the ZMS maturational stages did not influence the amount of protraction of the orbits. The 3D displacement of orbitale was influenced significantly by both the ZMS maturational stage and the type of therapy. ZMS stage B demonstrated a significantly greater 3D displacement of orbitale than ZMS stage $C$ $(0.7 \mathrm{~mm})$, while the BAMP group showed a significantly greater $3 \mathrm{D}$ displacement of orbitale compared to RME/FM group $(0.8 \mathrm{~mm})$. No significant influence of either type of therapy or maturational stage of ZMSs was assessed for the supero-inferior displacement of the orbits.

The antero-posterior displacement of first molars was influenced significantly by the type of therapy, with the BAMP group presenting with a significantly greater forward displacement of the first molar than the RME/FM group (1.3 mm - Table 7). No significant influence of either type of therapy or maturational stage of ZMSs was assessed for the supero-inferior displacement of the first molars.

\section{4 | DISCUSSION}

Previous experimental studies on monkeys ${ }^{17,19-21}$ have shown that the zygomaticomaxillary sutures and the pterygopalatine sutures 
TAB LE 4 Two-way ANOVA for the variable A point. The two factors tested were Treatment (BAMP and RME/FM) and the ZMS stages (A, B and C)

\begin{tabular}{|c|c|c|c|c|c|}
\hline \multicolumn{6}{|l|}{$\begin{array}{l}\text { Point A } \\
\text { Antero-posterior }\end{array}$} \\
\hline Source of variation & DF & SS & MS & $F$ & $P$ \\
\hline ZMS Stage & 2 & 12.918 & 6.459 & 5.978 & .006 \\
\hline Treatment & 1 & 30.525 & 30.525 & 28.251 & $<.001$ \\
\hline Total & 36 & 80.808 & 2.245 & & \\
\hline \multicolumn{6}{|c|}{ Comparisons for factor: ZMS Stage } \\
\hline Comparison & Diff. of means & $\mathrm{t}$ & $P$ & Critical level & Significant? \\
\hline \multicolumn{6}{|c|}{ Comparisons for factor: Treatment } \\
\hline Comparison & Diff. of means & $\mathrm{t}$ & $P$ & Critical level & Significant? \\
\hline BAMP vs RME/FM & 1.9 & 5.315 & .000 & 0.050 & Yes \\
\hline \multicolumn{6}{|l|}{ Supero-inferior } \\
\hline Source of variation & DF & SS & MS & $F$ & $P$ \\
\hline ZMS Stage & 2 & 6.207 & 3.103 & 2.140 & .135 \\
\hline Treatment & 1 & 11.120 & 11.120 & 7.666 & .009 \\
\hline ZMS Stage $x$ Treatment & 2 & 7.198 & 3.599 & 2.481 & .100 \\
\hline \multicolumn{6}{|l|}{$3 \mathrm{D}$} \\
\hline Source of variation & DF & SS & MS & $F$ & $P$ \\
\hline ZMS Stage & 2 & 6.052 & 3.026 & 3.191 & .055 \\
\hline Treatment & 1 & 12.607 & 12.607 & 13.293 & $<.001$ \\
\hline ZMS Stage $x$ Treatment & 2 & 1.182 & 0.591 & 0.623 & .543 \\
\hline Residual & 31 & 29.401 & 0.948 & & \\
\hline Total & 36 & 49.497 & 1.375 & & \\
\hline \multicolumn{6}{|c|}{ Comparisons for factor: Treatment } \\
\hline Comparison & Diff. of means & $\mathrm{t}$ & $P$ & Critical level & Significant? \\
\hline BAMP vs RME/FM & 1.2 & 3.646 & .001 & 0.050 & Yes \\
\hline
\end{tabular}

Differences of means were calculated on the least-square means $(\mathrm{mm})$.

DF, Degrees of Freedom; SS, sum of squares; MS, mean squares.

presented similar histological findings, with more complexity of interdigitations compared to other circummaxillary sutures. The ZMSs are the longest and largest of all circummaxillary sutures. ${ }^{17,18,21}$ In an earlier study, individual assessment of the maturation of the ZMSs was proposed as a possible indicator of the response to orthopaedic maxillary protraction in Class III patients. ${ }^{22}$ Clinically, maxillary protraction has been shown to be unpredictable with respect to chronological age at the start of treatment. ${ }^{2,8,11,13,24}$ 
TAB LE 5 Two-way ANOVA for the variable Zygoma Average. The two factors tested were Treatment (BAMP and RME/FM) and the ZMS stages (A, B and C)

\begin{tabular}{|c|c|c|c|c|c|}
\hline \multicolumn{6}{|l|}{$\begin{array}{l}\text { Zygoma average } \\
\text { Antero-posterior }\end{array}$} \\
\hline Source of variation & DF & SS & MS & $F$ & $P$ \\
\hline ZMS Stage & 2 & 2.423 & 1.211 & 1.473 & .245 \\
\hline Treatment & 1 & 7.802 & 7.802 & 9.488 & .004 \\
\hline ZMS Stage $x$ Treatment & 2 & 4.761 & 2.380 & 2.895 & .070 \\
\hline Total & 36 & 42.491 & 1.180 & & \\
\hline \multicolumn{6}{|c|}{ Comparisons for factor: Treatment } \\
\hline Comparison & Diff of means & $\mathrm{t}$ & $P$ & Critical level & Significant? \\
\hline ZMS Stage & 2 & 0.126 & 0.063 & 0.073 & .930 \\
\hline Treatment & 1 & 1.319 & 1.319 & 1.520 & .227 \\
\hline ZMS Stage $x$ Treatment & 2 & 5.945 & 2.973 & 3.425 & .051 \\
\hline Residual & 31 & 26.907 & 0.868 & & \\
\hline Total & 36 & 33.150 & 0.921 & & \\
\hline \multicolumn{6}{|l|}{ 3D } \\
\hline Source of variation & DF & SS & MS & $F$ & $P$ \\
\hline ZMS Stage & 2 & 2.351 & 1.175 & 2.474 & .101 \\
\hline Comparison & Diff of means & $\mathrm{t}$ & $P$ & Critical level & Significant? \\
\hline BAMP vs RME/FM & 0.9 & 3.953 & .000 & 0.050 & Yes \\
\hline
\end{tabular}

Differences of means were calculated on the least-square means.

DF, Degrees of Freedom; SS, sum of squares; MS, mean squares.

The present study showed that the stage of ZMS maturation was significantly associated with the amount of maxillary protraction. A significantly greater forward displacement of the maxilla (measured at Point A) was found when treatment with either BAMP or RME/FM was performed at ZMS maturational stages $A$ and $B$ compared to stage $C$ (1.3 mm and $1.4 \mathrm{~mm}$, respectively; Table 4). There was greater protraction of the orbits at orbitale in the BAMP group at ZMS maturational stage A vs stage $C(1.3 \mathrm{~mm})$ (Table 6). Stage $C$ demonstrated many bony bridges along the ZMS that presumably hampered the forward displacement of the maxilla and the orbits in the BAMP group, reflecting less maxillary protraction compared to stages $A$ and $B$ for both groups (Figures 1-4).

The type of therapy also influenced the response to maxillary protraction significantly. BAMP treatment promoted greater forward displacement of Point $A$, the zygomas, and the orbits than did RME/FM treatment (1.0-1.2 mm, Tables 4-6), corroborating the results of Hino et al. ${ }^{24}$ However, one of the limitation of these previous studies is that displacement measurements were made from iterative closest point (ICP). ICP does not distinguish between vertical and AP displacement nor does it record displacement of analogous landmarks. This study utilizes the methods described by Yatabe et al. ${ }^{29}$ to measure 3D displacements between analogous points and the $x, y$ and $z$ components of these 3D distances. It also incorporated standardized reference planes into the registered models. It is interesting to note that RME/FM therapy produced significantly greater displacement of Point $A$ in a downward direction with respect to the BAMP protocol (1.2 mm; Table 4).

According to chronological age, greater skeletal results would be expected in RME/FM patients, because this group was younger than the BAMP group at the start of treatment. Usually, BAMP has been 
recommended during the late mixed dentition or early permanent dentition or when the patient has reached 10 years of age $\mathrm{e}^{31,32}$ because most of the failures of zygomatic plates occurred in the youngest patients. ${ }^{16}$ The anchorage of BAMP essentially is skeletal and the orthopaedic forces are applied close to the circummaxillary sutures, increasing its orthopaedic effects.

TAB LE 6 Two-way ANOVA for the variable Orbitale Average. The two factors tested were Treatment (BAMP and RME/FM) and the ZMS stages (A, B and C)

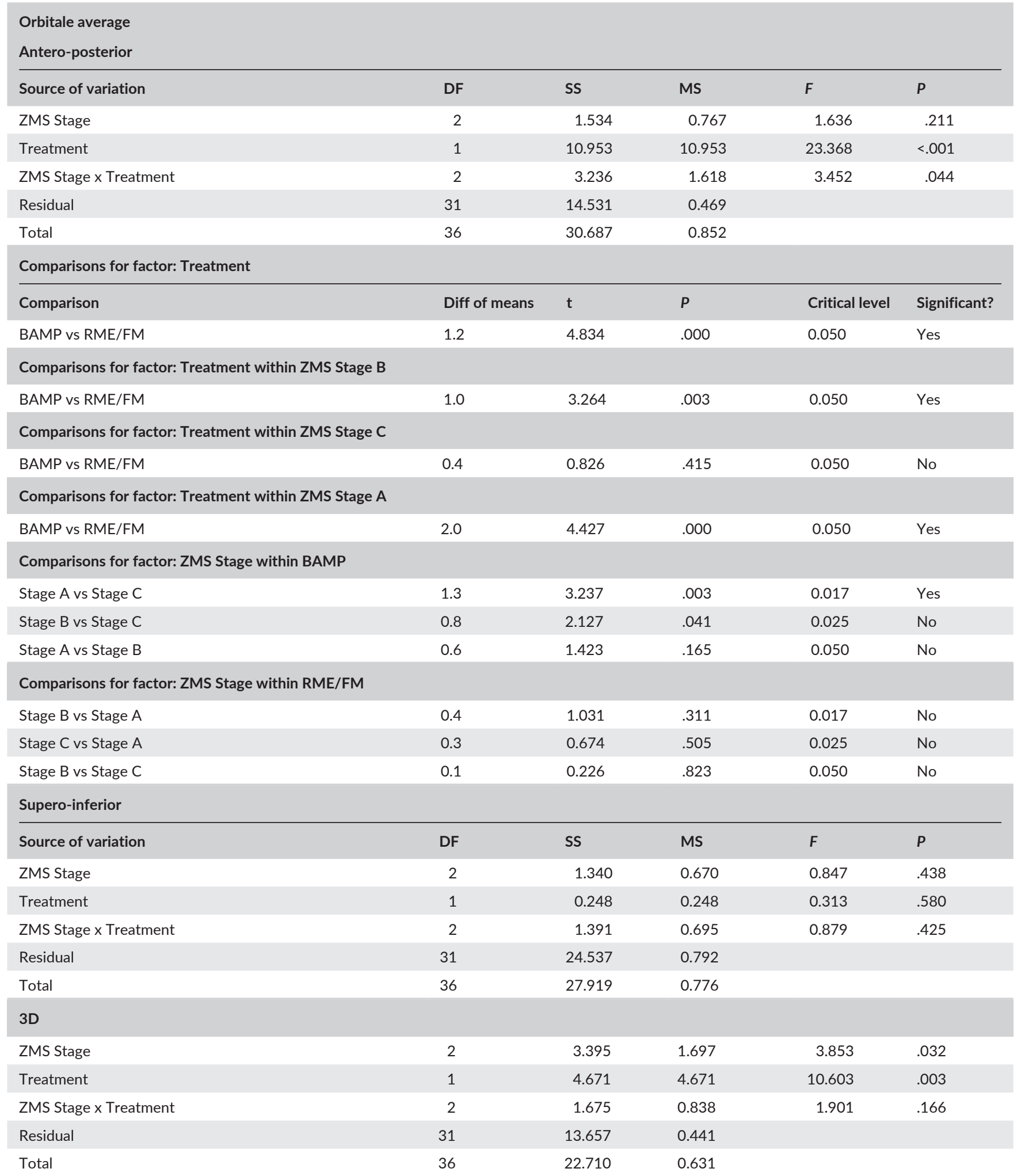


TABLE 6 (Continued)

\begin{tabular}{|c|c|c|c|c|c|}
\hline \multicolumn{6}{|c|}{ Comparisons for factor: ZMS Stage } \\
\hline Comparison & Diff of means & $\mathbf{t}$ & $P$ & Critical level & Significant? \\
\hline Stage B vs Stage C & 0.7 & 2.776 & .009 & 0.017 & Yes \\
\hline Stage A vs Stage C & 0.5 & 1.532 & .136 & 0.025 & No \\
\hline Stage B vs Stage A & 0.3 & 0.970 & .339 & 0.050 & No \\
\hline \multicolumn{6}{|c|}{ Comparisons for factor: Treatment } \\
\hline BAMP vs RME/FM & 0.8 & 3.256 & .003 & 0.050 & Yes \\
\hline
\end{tabular}

Differences of means were calculated on the least-square means ( $\mathrm{mm})$.

DF, Degrees of Freedom; SS, sum of squares; MS, mean squares.

Despite the higher force delivered by RME/FM treatment, the force is applied to the expander attached to the maxillary teeth dissipating the force to the periodontal ligament area. ${ }^{31,33}$ Probably, because of that, the ZMS maturational stages did not influence the quantity of the protraction of the orbits in the RME/FM group (Table 5). In addition, it should be noted that the response to maxillary protraction is highly dependent on patient cooperation. Patient compliance tends to be lower in patients with a facial mask compared to BAMP with Class III elastics. ${ }^{31}$

While the ZMS maturational stages and type of therapy may influence the amount and/or direction of maxillary protraction, the interaction between these factors was not statistically significant for the antero-posterior displacement of Point A (Table 4). This finding probably can be explained by a significantly greater maxillary protraction with the BAMP protocol compared to the RME/FM protocol only in patients at ZMS Stages A or B. No significant differences between the two therapies were found in the amount of maxillary protraction for patients with ZMS Stage C. On the other hand, there was a significant interaction between ZMS maturational stages and type of therapy in the forward displacement of the orbits. The BAMP group demonstrated greater forward displacement of the orbits at ZMS maturational stages $A$ and $B$ with respect to RME/FM $(2.0 \mathrm{~mm}$ and $1.0 \mathrm{~mm}$, respectively; Table 6).

The ZMS were evaluated on both sides and, when they presented different maturational stages, the more mature stage of ZMSs was considered. One of these cases presented ZMS stage B on the right side and $A$ on the left. In this patient, probably the clinical difference in

TAB LE 7 Two-way ANOVA for the variable First Molar Average. The two factors tested were Treatment (BAMP and RME/FM) and the ZMS stages (A, B and C).

\begin{tabular}{|c|c|c|c|c|c|}
\hline \multicolumn{6}{|l|}{$\begin{array}{l}\text { First molar average } \\
\text { Antero-posterior }\end{array}$} \\
\hline Source of variation & DF & SS & MS & $F$ & $P$ \\
\hline ZMS Stage & 2 & 13.232 & 6.616 & 2.310 & .116 \\
\hline Treatment & 1 & 13.552 & 13.552 & 4.732 & .037 \\
\hline ZMS Stage $x$ Treatment & 2 & 14.491 & 7.246 & 2.530 & .096 \\
\hline Total & 36 & 127.494 & 3.541 & & \\
\hline \multicolumn{6}{|c|}{ Comparisons for factor: Treatment } \\
\hline Comparison & Diff of means & $\mathrm{t}$ & $P$ & Critical level & Significant? \\
\hline BAMP vs RME/FM & 1.3 & 2.175 & .037 & 0.050 & Yes \\
\hline ZMS Stage & 2 & 2.447 & 1.224 & 0.614 & .548 \\
\hline Treatment & 1 & 0.111 & 0.111 & 0.055 & .815 \\
\hline ZMS Stage $x$ Treatment & 2 & 12.407 & 6.203 & 3.111 & .059 \\
\hline Residual & 31 & 61.819 & 1.994 & & \\
\hline Total & 36 & 77.930 & 2.165 & & \\
\hline
\end{tabular}

Differences of means were calculated on the least-square means (mm).

DF, Degrees of Freedom; SS, sum of squares; MS, mean squares. 

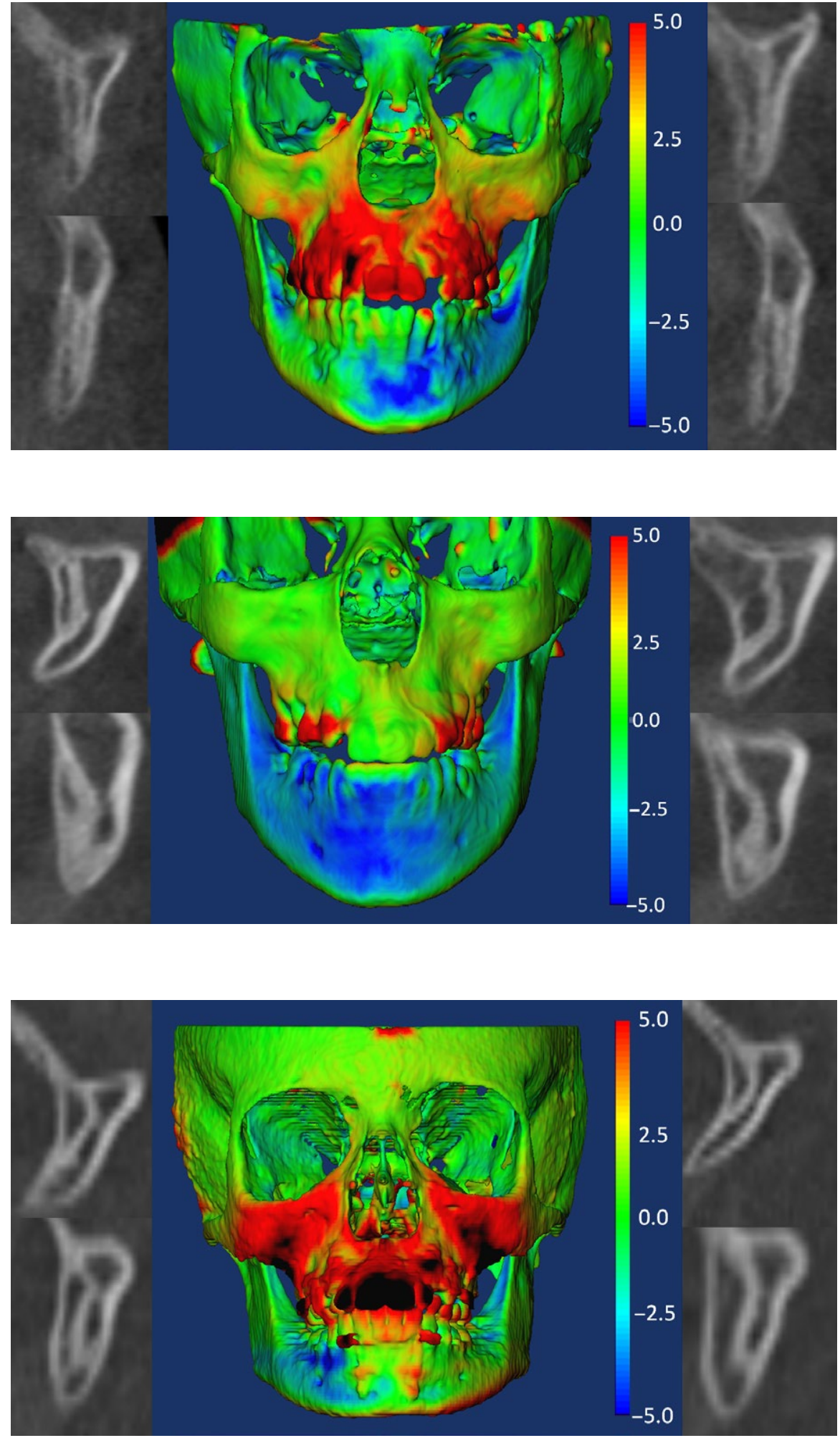

FIGURE 1 Patient with good response from rapid maxillary expansion and facial mask treatment and zygomaticomaxillary sutures at stage B. Colour-coded map is used for visualization of the maxillary protraction and graphic display of the 3D surface distances in $\mathrm{mm}$ [Colour figure can be viewed at wileyonlinelibrary.com]

FIGURE 2 Patient in the rapid maxillary expansion and facial mask group who presented poor response to maxillary protraction. Note that zygomaticomaxillary sutures were at Stage C. Colour-coded map is used for visualization purposes and graphic display of the 3D surface distances in $\mathrm{mm}$ [Colour figure can be viewed at wileyonlinelibrary.com]

FIGURE 3 Patient with

zygomaticomaxillary sutures at Stage A and marked response of maxillary and zygomatic protraction with bone-anchored maxillary protraction treatment. Colourcoded map is used for visualization purposes and graphic display of the $3 D$ surface distances in $\mathrm{mm}$ [Colour figure can be viewed at wileyonlinelibrary.com] response to treatment would be insignificant as the maxillary protraction showed a very satisfactory result on both sides. Another patient who presented with ZMS stage $B$ on the right side and stage $C$ on the left had a very poor protraction effect of the maxilla. It is possible that the ZMS stage $C$ hampered the maxillary protraction on both sides. For these cases, we considered the more mature maturational stage of ZMS for the statistical analysis. However, future investigations are needed to evaluate the clinical importance of a one-sided fusion or more advanced maturational stage of the ZMS.

The CBCT images of three patients showing ZMS maturational stage $D$ (one female and one male patient, both 12.5 years of age) or stage $E$ (a 13-year-old male patient) were analysed but were removed from the original BAMP sample for statistical reasons. It is interesting to note that these patients did not exhibit much maxillary protraction, with 
FIGURE 4 Patient in the boneanchored maxillary protraction group who presented poor response to maxillary protraction. Note that zygomaticomaxillary sutures were at Stage C. Colour-coded map is used for visualization purposes and graphic display of the 3D surface distances in $\mathrm{mm}$ [Colour figure can be viewed at wileyonlinelibrary.com]
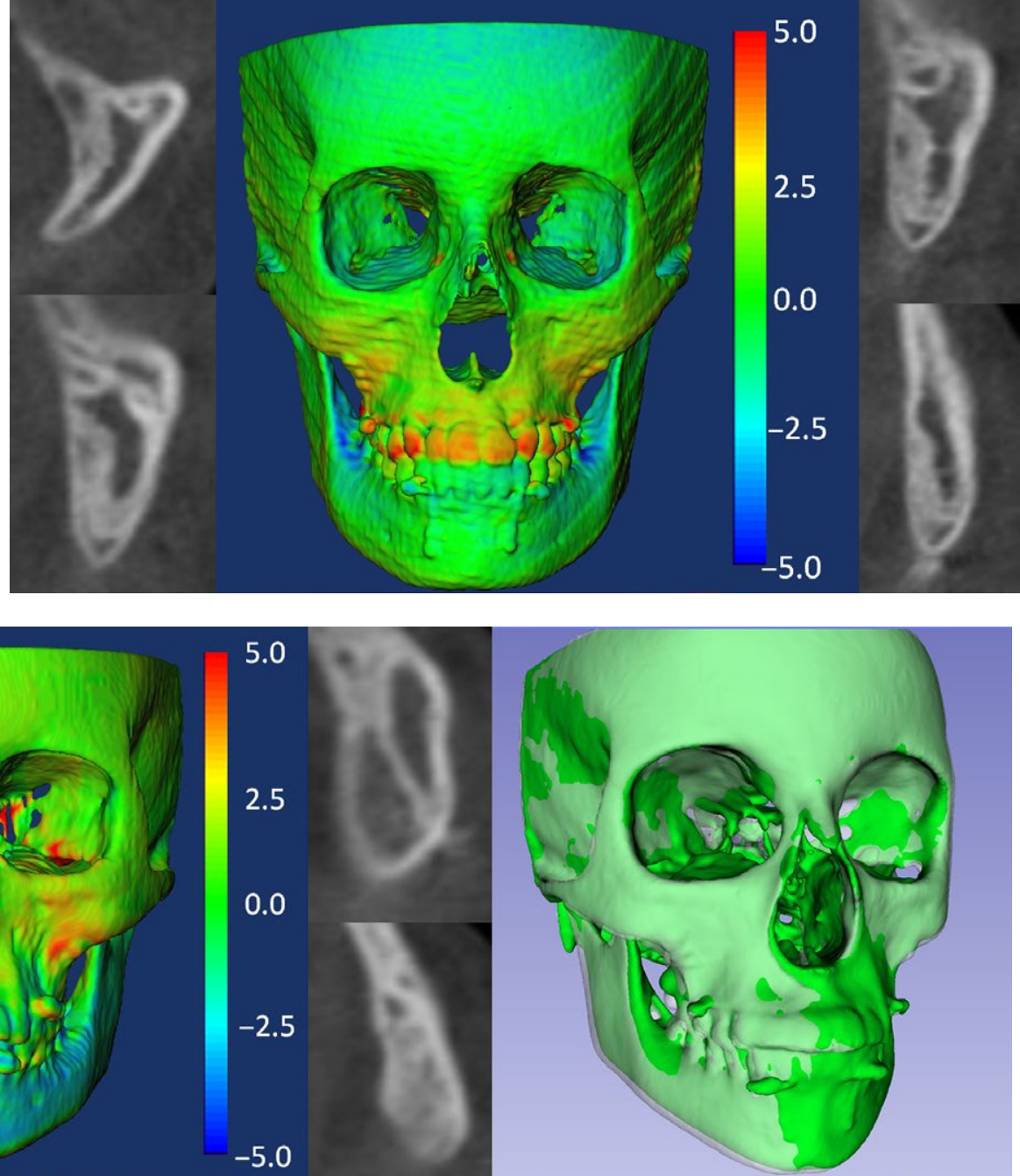

FIGURE 5 Patient at Stage D of zygomaticomaxillary sutures maturation, who presented mostly vertical response to maxillary protraction with bone-anchored maxillary protraction, as can be seen in the semi-transparent overlay in the right. Colour-coded map is used for visualization purposes and graphic display of the 3D surface distances in $\mathrm{mm}$. In semi-transparent overlay in the right, the time 1 surface model is shown in green and the time 2 is shown in transparent white [Colour figure can be viewed at wileyonlinelibrary.com]
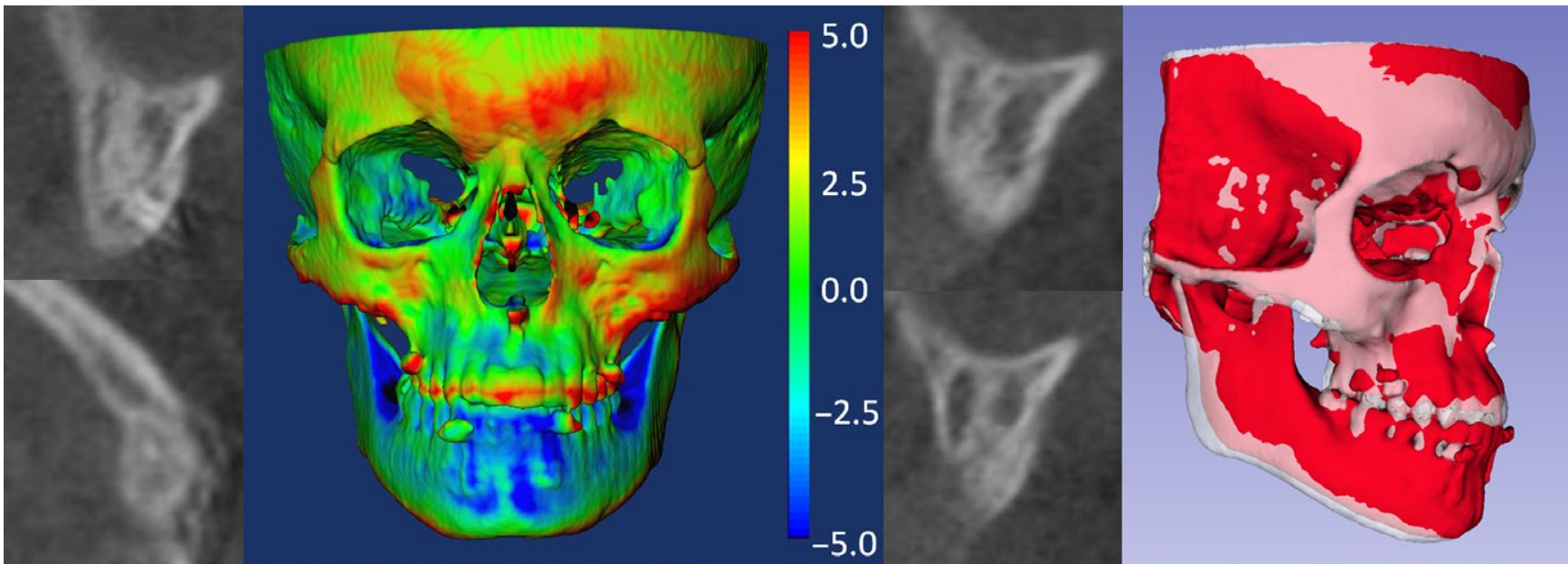

FIGURE 6 Boy with zygomaticomaxillary sutures at Stage D demonstrating only little vertical effect after bone-anchored maxillary protraction treatment. Colour-coded map is used for visualization purposes and graphic display of the 3D surface distances in mm. In semitransparent overlay in the right, the time 1 surface model is shown in red and the time 2 is shown in transparent white [Colour figure can be viewed at wileyonlinelibrary.com] 


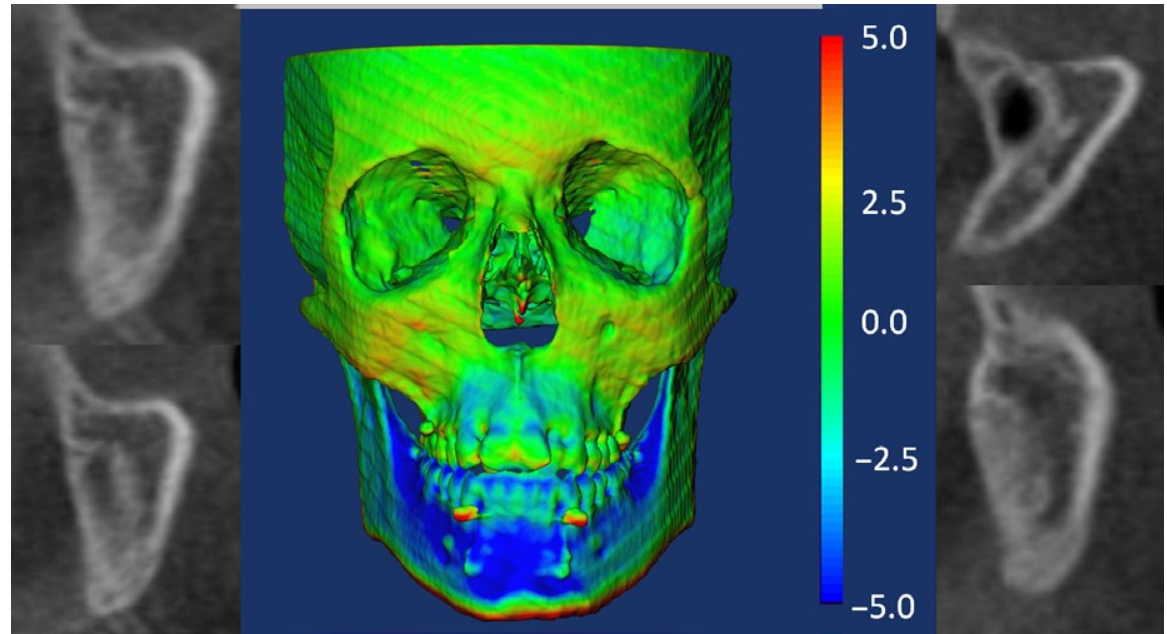

FIGURE 7 Patient at Stage E of zygomaticomaxillary sutures maturation, who presented almost no response to maxillary protraction with bone-anchored maxillary protraction. Colour-coded map is used for visualization purposes and graphic display of the 3D surface distances in $\mathrm{mm}$ [Colour figure can be viewed at wileyonlinelibrary.com] both patients at stage $\mathrm{D}$ presenting an antero-posterior displacement of the maxilla of about $-0.5 \mathrm{~mm}$, with no sagittal effect at the zygomas and orbits. The main effect was in a vertical direction, with an inferior displacement of maxilla of $1.5 \mathrm{~mm}$ and $1.7 \mathrm{~mm}$ (Figures 5 and 6).

Interestingly, the patient at stage $\mathrm{E}$ showed no sagittal or vertical effect in maxilla position $(-1.2 \mathrm{~mm}$ and $-0.2 \mathrm{~mm}$, respectively) or the position of the orbits, with only inferior displacement of the zygomas (1.6 mm; Figure 7) observed. In this patient, the BAMP protocol seemed to produce some vertical skeletal effects while presenting with a more mature ZMS stage. This preliminary finding needs to be confirmed in samples of patients with more mature ZMS stages.

This study applied a validated classification of maturation of ZMS to individually assess the morphology of this circummaxillary suture prior to orthopaedic maxillary protraction, mainly for juvenile and early adolescent patients for whom this treatment still is unpredictable. This method potentially can predict a good response from RME/FM treatment for patients at stages $A$ or $B$ up to 15 years of age or avoid the failure and side effects of the BAMP or RME/FM treatments for younger patients showing fusion of ZMSs. However, the sample size for some maturational stages of ZMS was relatively small in the present study, and future investigations of stages $D$ and $E$, in which the ZMSs are fused, may further elucidate the clinical responses at each ZMS stage.

Thus, the acquisition of a pre-treatment small field CBCT image including the ZMS region may be used as a triage tool for prediction of the orthopaedic result for BAMP and RME/FM as well to evaluate bone quality and thickness in the infrazygomatic crest for BAMP placement, at a relatively low radiation dose. Future studies, however, would be recommended using a larger sample to confirm our results prior the application of the present classification method in the clinical practice.

\section{5 | CONCLUSIONS}

The early maturational stages of the ZMS are related directly to the greater amount of maxillary protraction response resulting from RME/ FM or BAMP treatments. Class III patients with ZMS stages A and B demonstrated greater maxillary protraction than patients at stage $C$ (Point $\mathrm{A}$ and orbitale region).

\section{ACKNOWLEDGEMENTS}

The authors thank Hugo J. De Clerck and Claudia Toyama Hino for allowing the examination of samples of BAMP and facial mask.

\section{REFERENCES}

1. Baccetti T, Franchi L, McNamara JA Jr. Treatment and posttreatment craniofacial changes after rapid maxillary expansion and facemask therapy. Am J Orthod Dentofacial Orthop. 2000;118:404-413.

2. Baik HS. Clinical results of the maxillary protraction in Korean children. Am J Orthod Dentofacial Orthop. 1995;108:583-592.

3. Franchi L, Baccetti T, McNamara JA Jr. Shape-coordinate analysis of skeletal changes induced by rapid maxillary expansion and facial mask therapy. Am J Orthod Dentofacial Orthop. 1998;114:418-426.

4. Ngan P, Hägg U, Yiu C, Merwin D, Wei SH. Soft tissue and dentoskeletal profile changes associated with maxillary expansion and protraction headgear treatment. Am J Orthod Dentofacial Orthop. 1996;109:438-459.

5. Macdonald KE, Kapust AJ, Turley PK. Cephalometric changes after the correction of class III malocclusion with maxillary expansion/facemask therapy. Am J Orthod Dentofacial Orthop. 1999;116:13-24.

6. Ngan P, Yiu C, Hu A, Hägg U, Wei SH, Gunel E. Cephalometric and occlusal changes following maxillary expansion and protraction. Eur J Orthod. 1998;20:237-254.

7. Cordasco G, Matarese G, Rustico L, et al. Efficacy of orthopedic treatment with protraction facemask on skeletal Class III malocclusion: a systematic review and meta-analysis. Orthod Craniofac Res. 2014;17:133-143.

8. Hickham JH. Maxillary protraction therapy: diagnosis and treatment. J Clin Orthod. 1991;25:102-113.

9. Proffit W, Fields H, Sarver D. Contemporary Orthodontics. 5th ed. St Louis: CV Mosby; 2012.

10. Wells AP, Sarver DM, Proffit WR. Long-term efficacy of reverse pull headgear therapy. Angle Orthod. 2006;76:915-922.

11. Kapust AJ, Sinclair PM, Turley PK. Cephalometric effects of facemask/ expansion therapy in Class III children: a comparison of three age groups. Am J Orthod Dentofacial Orthop. 1998;113:204-212.

12. Franchi L, Baccetti T, McNamara JA Jr. Postpubertal assessment of treatment timing for maxillary expansion and protraction 
therapy followed by fixed appliances. Am J Orthod Dentofacial Orthop. 2004;126:555-568.

13. Yüksel S, Uçem TT, Keykubat A. Early and late facemask therapy. Eur J Orthod. 2001;23:559-568.

14. Yavuz I, Halicioğlu K, Ceylan I. Face mask therapy effects in two skeletal maturation groups of female subjects with skeletal Class III malocclusions. Angle Orthod. 2009;79:842-848.

15. Nguyen T, Cevidanes LHS, Cornelis MA, Heymann GC, Paula LK, De Clerck HJ. Three-dimensional assessment of maxillary changes associated with bone anchored maxillary protraction. Am J Orthod Dentofacial Orthop. 2011;140:790-798.

16. Cornelis MA, Scheffler NR, Mahy P, Siciliano S, De Clerck HJ, Tulloch JF. Modified miniplates for temporary skeletal anchorage in orthodontics: placement and removal surgeries. J Oral Maxillofac Surg. 2008;66:1439-1445.

17. Kambara T. Dentofacial changes produced by extraoral forward force in the Macaca irus. Am J Orthod. 1977;71:249-277.

18. Sholts SB, Wärmländer SKTS. Zygomaticomaxillary suture shape analyzed with digital morphometrics: reassessing patterns of variation in American Indian and European populations. Forensic Sci Int. 2012;217:234e. 231-234e.236

19. Jackson G, Kokich V, Shapiro P. Experimental and post-experimental response to anteriorly directed extraoral force in young Macaca nemestrina. Am J Orthod. 1979;75:318-333.

20. Zhao N, Xu Y, Chen Y, Xu Y, Han X, Wang L. Effects of Class III magnetic orthopedic forces on the craniofacial sutures of rhesus monkeys. Am J Orthod Dentofacial Orthop. 2008;133:401-409.

21. Nanda R, Hickory W. Zygomaticomaxillary suture adaptations incident to anteriorly-directed forces in rhesus monkeys. Angle Orthod. 1984;54:199-210.

22. Angelieri F, Franchi L, Cevidanes LHS, Toyama-Hino C, Nguyen T, McNamara JA Jr. Zygomaticomaxillary suture maturation: a predictor of maxillary protraction? Part I: classification method. Orthod Craniofac Res. 2017;20:85-94.

23. Turley PK. Orthopedic correction of Class III malocclusion with palatal expansion and custom protraction headgear. J Clin Orthod. 1988;22:314-325.

24. Hino CT, Cevidanes LH, Nguyen TT, De Clerck HJ, Franchi L, McNamara JA Jr. Three-dimensional analysis of maxillary changes associated with facemask and rapid maxillary expansion compared with bone anchored maxillary protraction. Am J Orthod Dentofacial Orthop. 2013;144:705-714.
25. Cevidanes LH, Heymann G, Cornelis MA, De Clerck HJ, Tulloch JF. Superimposition of 3-dimensional cone-beam computed tomography models of growing patients. Am J Orthod Dentofacial Orthop. 2009;136:94-99.

26. Ruellas AC, Yatabe MS, Gomes M, et al. Common 3D coordinate system for assessments of directional changes. Am J Orthod Dentofacial Orthop. 2016;149:645-656.

27. Ruellas AC, Huanca Ghislanzoni LT, Gomes MR, et al. Comparison and reproducibility of 2 regions of reference for maxillary regional registration with cone-beam computed tomography. Am J Orthod Dentofacial Orthop. 2016;149:533-542.

28. Ruellas ACO, Yatabe MS, Souki BQ, et al. 3D mandibular superimposition: comparison of regions of reference for voxel-based registration. PLoS ONE. 2016;11:e0157625.

29. Yatabe M, Garib DG, Faco RAS, et al. Mandibular and glenoid fossa changes after bone anchored maxillary protraction in patients with UCLP: a 3-D preliminary assessment. Angle Orthod. 2017;87(3):423-431. doi: 10.2319/052516-419.1

30. Faul F, Erdfelder E, Lang A-G, Buchner A. G*Power 3: a flexible statistical power analysis program for the social, behavioral, and biomedical sciences. Behav Res Methods. 2007;39:175-191.

31. Cevidanes LHS, Baccetti T, Franchi L, McNamara JA Jr, De Clerck HJ. Comparison of two protocols for maxillary protraction: bone anchors versus face mask with rapid maxillary expansion. Angle Orthod. 2010;80:799-806.

32. De Clerck HJ, Cevidanes LHS, Baccetti T. Dentofacial effects of bone-anchored maxillary protraction: a controlled study on consecutively treated Class III patients. Am J Orthod Dentofacial Orthop. 2010;138:577-581.

33. Kircelli BH, Pektas ZO. Midfacial protraction with skeletally anchored face mask therapy: a novel approach and preliminary results. Am J Orthod Dentofacial Orthop. 2008;133:440-449.

How to cite this article: Angelieri F, Ruellas AC, Yatabe MS, et al. Zygomaticomaxillary suture maturation: Part II-The influence of sutural maturation on the response to maxillary protraction. Orthod Craniofac Res. 2017;20:152-163. https://doi.org/10.1111/ocr.12191 